Internat. J. Math. \& Math. Sci.

Vol. 22, No. 1 (1999) 67-74

S $0161-1712\langle 99\rangle 22067-4$

(c) Electronic Publishing House

\title{
NONCOMMUTATIVITY AND NONCENTRAL ZERO DIVISORS
}

\author{
HOWARD E. BELL and ABRAHAM A. KLEIN
}

(Received 11 September 1997)

\begin{abstract}
Let $R$ be a ring, $Z$ its center, and $D$ the set of zero divisors. For finite noncommutative rings, it is known that $D \backslash Z \neq \varnothing$. We investigate the size of $|D \backslash Z|$ in this case and, also, in the case of infinite noncommutative rings with $D \backslash Z \neq \varnothing$.
\end{abstract}

Keywords and phrases. Finite noncommutative rings, infinite noncommutative rings, central and noncentral zero divisors.

1991 Mathematics Subject Classification. 16P10, 16U99.

It has been known for many years that for certain classes of rings, commutativity or noncommutativity is determined by the behavior of zero divisors or nilpotent elements. Among the early theorems illustrating this phenomenon are two due to Herstein, the second of which is obviously an extension of the first.

THEOREM $H_{1}$ [4]. If $R$ is a finite ring in which all zero divisors are central, then $R$ is commutative.

THEOREM $\boldsymbol{H}_{2}$ [5]. If $R$ is a periodic ring in which all nilpotent elements are central, then $R$ is commutative.

From these results we know that a periodic ring which is not commutative must contain noncentral nilpotent elements. We first consider the question of how large the set of noncentral zero divisors must be in a finite noncommutative ring, and then we comment on some related questions for infinite rings. In our final section, we establish the commutativity of certain rings in which appropriate subsets of nonnilpotent zero divisors are assumed to be central.

1. Preliminaries. In general, $R$ represents a ring, not necessarily with 1 , and $Z$ its center. For $Y$ an element or subset of $R, A(Y)$ is the two-sided annihilator of $Y$; and for $H \subseteq R,|H|$ denotes the cardinal number of $H$. The symbols $N=N(R), D=D(R)$, and $S=S(R)$ denote, respectively, the set of nilpotent elements, the set of zero divisors, and the set of zero divisors $a$ for which $A(a) \neq\{0\}$.

In several of our proofs, it is necessary to show that certain sums of zero divisors are zero divisors. The following lemma is helpful.

LEMMA 1.1. Let $R$ be any ring.

(i) If $a \in N, b \in D$ and $a b=b a$, then $a+b \in D$. Moreover, $a+b \in N$ if and only if $b \in N$.

(ii) If $a \in N, b \in S$ and $a b=b a$, then $a+b \in S$.

Proof. (i) We may assume that $b \in D$ is a left zero divisor, and choose $c \neq 0$ 
such that $b c=0$. Let $k \geq 1$ be the minimal positive integer for which $a^{k} c=0$. Then $(a+b) a^{k-1} c=a^{k} c+a^{k-1} b c=0$, so $a+b \in D$. Finally, if $a+b \in N$, then $b=a+b-a \in$ $N$ since $a+b$ and $a$ commute.

(ii) Let $b c=c b=0, c \neq 0$. Choose $k$ such that $a^{k} c=0 \neq a^{k-1} c$ and choose $j$ such that $a^{k-1} c a^{j}=0 \neq a^{k-1} c a^{j-1}$. Then $a^{k-1} c a^{j-1}(a+b)=0=(a+b) a^{k-1} c a^{j-1}$, and hence $a+b \in S$.

2. $|D \backslash Z|$ in finite rings. Our first theorem appears in [6]. However, the proof we present is very different from, and more elementary than, the proof given in [6]; and it will shed light on a later question.

THEOREM 2.1. Let $R$ be a finite indecomposable noncommutative ring in which $|R|$ is a power of the prime $p$. Then $|D \backslash Z| \geq\left(p^{2}-1\right)|D \cap Z|$.

Proof. If $R=D$, then the fact that $[R: Z]$ cannot be $p$ gives $[R: Z] \geq p^{2}$; and we get $|D \backslash Z|=|R \backslash Z| \geq\left(p^{2}-1\right)|Z|=\left(p^{2}-1\right)|D \cap Z|$. Thus, we may assume henceforth that $R \neq D$, in which case $R$ has 1 and all elements of $R \backslash D$ are invertible.

Observe that by Lemma 1.1(i), if $y \in D$ and $u \in N \cap Z$, then $y+u \in D$. Also, observe that, by the indecomposability of $R$, there cannot be any nonzero central idempotent zero divisiors in $R$; hence, $D \cap Z \subseteq N$.

Suppose that $D \neq N$. Since every element of $R$ has an idempotent power, there must exist a noncentral idempotent $e \in D$. For some $x \in R,[e, x] \neq 0$, so either $(1-e) x e$ or $e x(1-e)$ is noncentral. Assume the latter, let $u=e x(1-e)$, and let $f=e+u$. Then $f$ is another idempotent; and $e f=f, f e=e, e u=f u=u$, and $u e=u f=u^{2}=0$. It follows by Lemma 1.1(i) that for all $i, j=0,1, \ldots, p-1$ with $(i, j) \neq(0,0), i e+j f+D \cap$ $Z \subseteq D \backslash Z$. Moreover, if $\left(i_{1}, j_{1}\right) \neq\left(i_{2}, j_{2}\right)$, then $\left(i_{1} e+j_{1} f+D \cap Z\right) \cap\left(i_{2} e+j_{2} f+D \cap Z\right)=$ $\varnothing$. Therefore, $|D \backslash Z| \geq\left(p^{2}-1\right)|D \cap Z|$.

Now suppose that $D=N$, in which case each element of $R$ is either nilpotent or invertible-a condition which implies that $N$ is an ideal [8] (in particular, that $N$ is closed under addition). Therefore, if there exist noncommuting $u_{1}, u_{2} \in N$, then for each $i, j=0, \ldots, p-1$ with $(i, j) \neq(0,0), i u_{1}+j u_{2}+D \cap Z \subseteq D \backslash Z$; and, as before, we have $p^{2}-1$ pairwise disjoint subsets of $D \backslash Z$, each of cardinality $|D \cap Z|$.

It remains only to consider the case of $D=N$ and $N$ commutative. Theorem $\mathrm{H}_{2}$ guarantees that $N \nsubseteq Z$, so there exists a noncentral $u \in N$ and an invertible $x \in R$ such that $[x, u] \neq 0$. In this case, the sets $i u+j x u+D \cap Z$ with $i, j=0,1, \ldots, p-1$ and $(i, j) \neq(0,0)$ provide $p^{2}-1$ pairwise disjoint subsets of $D \backslash Z$ with cardinality $|D \cap Z|$. To see this, note that if these sets contain central elements or fail to be pairwise disjoint, then there exist $i, j \in\{0,1, \ldots, p-1\}$ with $(i, j) \neq(0,0)$ such that $i u+j x u \in Z$. If either $i$ or $j$ is 0 , we have the contradiction $[x, u]=0$. Otherwise, we have $[i u+j x u, x]=0$, which may be rewritten as $(j x+i)[u, x]=0$. Thus, $j x+i \in$ $D=N$. Since $N$ is commutative, this gives the contradiction $[u, x]=0$. Our proof is now complete.

Theorem 2.1 is the major step in the proof of the following more general theorem. The remaining part is a simple lemma given in [6], which we need not repeat.

THEOREM 2.2 [6, Thm. 3.4]. If $R$ is a finite noncommutative ring, then there exists 
a prime $p$ such that $|D \backslash Z| \geq\left(p^{2}-1\right)|D \cap Z|$. In particular, $|D \backslash Z| \geq 3|D \cap Z|$.

Frequently, the noncentral zero divisors are more abundant than Theorem 2.2 states. We have

THEOREM 2.3. Let $R$ be a finite noncommutative ring with 1 ; and write $R=S_{1} \oplus S_{2} \oplus$ $\cdots \oplus S_{k}$, where the $S_{i}$ are indecomposable. If $n$ of the $S_{i}$ 's are noncommutative, then $|D \backslash Z| \geq\left(4^{n}-1\right)|D \cap Z|$, and the bound $4^{n}-1$ is best possible.

Proof. For $n=1$, the result holds by the previous theorem. Proceeding from $n$ to $n+1$, we may assume that $R=R_{1} \oplus R_{2}$, where $R_{1}$ is the sum of $n$ noncommutative $S_{j}$ and $R_{2}$ is the sum of the other $S_{j}$. Let $Z_{i}=Z\left(R_{i}\right)$ and $D_{i}=D\left(R_{i}\right), i=1,2$. Note that an element $\left(a_{1}, a_{2}\right) \in R$ with $a_{i} \in R_{i}, i=1,2$, is central (invertible) if and only if $a_{1}$ and $a_{2}$ have the same property in $R_{1}$ and $R_{2}$. By the induction hypothesis, $\left|D_{1} \backslash Z_{1}\right| \geq$ $\left(4^{n}-1\right)\left|D_{1} \cap Z_{1}\right|$; and $\left|D_{2} \backslash Z_{2}\right| \geq 3\left|D_{2} \cap Z_{2}\right|$.

Now $\left(a_{1}, a_{2}\right) \in D \cap Z$ if and only if $a_{2} \in D_{2} \cap Z_{2}$ and $a_{1} \in Z_{1}$ or $a_{2} \in Z_{2} \backslash D_{2}$ and $a_{1} \in D_{1} \cap Z_{1}$. Thus, we have

$$
|D \cap Z|=\left|Z_{1}\right|\left|D_{2} \cap Z_{2}\right|+\left|D_{1} \cap Z_{1}\right|\left|Z_{2} \backslash D_{2}\right| .
$$

The partition $R_{2}=\left(D_{2} \cap Z_{2}\right) \biguplus\left(Z_{2} \backslash D_{2}\right) \biguplus\left(D_{2} \backslash Z_{2}\right) \biguplus\left(R_{2} \backslash\left(D_{2} \cup Z_{2}\right)\right)$ yields four disjoint possibilities for $\left(a_{1}, a_{2}\right)$ to be in $D \backslash Z$; and we easily determine that

$$
|D \backslash Z|=\left|R_{1} \backslash Z_{1}\right|\left|D_{2} \cap Z_{2}\right|+\left|D_{1} \backslash Z_{1}\right|\left|Z_{2} \backslash D_{2}\right|+\left|R_{1}\right|\left|D_{2} \backslash Z_{2}\right|+\left|D_{1}\right|\left|R_{2} \backslash\left(D_{2} \cup Z_{2}\right)\right| .
$$

Now $\left[R_{2}: Z_{2}\right]$ cannot be prime. Thus, $\left[R_{2}: Z_{2}\right] \geq 4$. Since $R_{1}$ is the sum of $n$ noncommutative $S_{j}$, we have $\left[R_{1}: Z_{1}\right] \geq 4^{n}$.

We now consider the group of units in $R_{2}$, namely, $R_{2} \backslash D_{2}$. The subgroup $Z_{2} \backslash D_{2}$ is proper, since for $a \in N\left(R_{2}\right) \backslash Z_{2}$, we have $1+a \in\left(R_{2} \backslash D_{2}\right) \backslash\left(Z_{2} \backslash D_{2}\right)=R_{2} \backslash\left(D_{2} \cup Z_{2}\right)$. Since the complement of a proper subgroup has cardinality at least that of the subgroup, then setting $\left|Z_{2} \backslash D_{2}\right|=\alpha$ and $\left|R_{2} \backslash\left(D_{2} \cup Z_{2}\right)\right|=\beta$, we have $\beta=\alpha, \beta=2 \alpha$, or $\beta \geq$ $3 \alpha$. Since $\left|R_{2} \backslash Z_{2}\right| \geq 3\left|Z_{2}\right|$, we have $\left|D_{2} \backslash Z_{2}\right|+\beta \geq 3\left(\left|D_{2} \cap Z_{2}\right|+\alpha\right)$. Hence, $\left|D_{2} \backslash Z_{2}\right| \geq$ $3\left|D_{2} \cap Z_{2}\right|+(3-t) \alpha$ with $t=1$ when $\beta=\alpha, t=2$ when $\beta=2 \alpha$, and $t=3$ when $\beta \geq 3 \alpha$, the last case following from Theorem 2.2. Therefore,

$$
\begin{aligned}
\left|R_{1} \backslash Z_{1}\right|\left|D_{2} \cap Z_{2}\right| & +\left|R_{1}\right|\left|D_{2} \backslash Z_{2}\right| \\
& \geq\left(4^{n}-1\right)\left|Z_{1}\right|\left|D_{2} \cap Z_{2}\right|+4^{n}\left|Z_{1}\right|\left(3\left|D_{2} \cap Z_{2}\right|+(3-t) \alpha\right) \\
& \geq\left(4^{n+1}-1\right)\left|Z_{1}\right|\left|D_{2} \cap Z_{2}\right|+4^{n}(3-t)\left|Z_{1}\right| \alpha \\
& \geq\left(4^{n+1}-1\right)\left|Z_{1}\right|\left|D_{2} \cap Z_{2}\right|+4^{n}(3-t)\left|D_{1} \cap Z_{1}\right|\left|Z_{2} \backslash D_{2}\right|
\end{aligned}
$$

and

$$
\begin{aligned}
\left|D_{1} \backslash Z_{1}\right|\left|Z_{2} \backslash D_{2}\right|+\left|D_{1}\right| \mid R_{2} \backslash & \left(D_{2} \cup Z_{2}\right) \mid \\
& \geq\left(4^{n}-1\right)\left|D_{1} \cap Z_{1}\right|\left|Z_{2} \backslash D_{2}\right|+4^{n} t\left|D_{1} \cap Z_{1}\right|\left|Z_{2} \backslash D_{2}\right| .
\end{aligned}
$$

It now follows from (2.1)-(2.4) that $|D \backslash Z| \geq\left(4^{n+1}-1\right)|D \cap Z|$. 
To show that the bound $4^{n}-1$ is best possible, let $R=S_{1} \oplus \cdots \oplus S_{n}$, where

$$
S_{1}=\cdots=S_{n}=\left\{\left[\begin{array}{lll}
a & b & d \\
0 & a & c \\
0 & 0 & a
\end{array}\right] \mid a, b, c, d \in \mathrm{GF}(2)\right\} .
$$

Since the center of $S_{i}$ is

$$
\left\{\left[\begin{array}{lll}
a & 0 & d \\
0 & a & 0 \\
0 & 0 & a
\end{array}\right] \mid a, d \in \mathrm{GF}(2)\right\}
$$

and it has two invertible elements, we have $|D \cap Z|=|Z \backslash(Z \backslash D)|=4^{n}-2^{n}=2^{n}\left(2^{n}-1\right)$. Now the invertible elements of $S_{i}$ are those matrices with $a=1$, so

$$
|D|=|R \backslash(R \backslash D)|=16^{n}-8^{n}=8^{n}\left(2^{n}-1\right)=4^{n}|D \cap Z|
$$

and

$$
|D \backslash Z|=\left(4^{n}-1\right)|D \cap Z| .
$$

REMARK. If $p$ is the smallest prime dividing $|R|$, then $4^{n}-1$ may be replaced by $p^{2 n}-1$ in Theorem 2.3 .

Theorem $\mathrm{H}_{2}$ suggests that there may be analogues of Theorems 2.2 and 2.3 with $D$ replaced by $N$, and indeed there are.

THEOREM 2.4. If $R$ is a finite noncommutative ring and $p$ is the smallest prime dividing $|R|$, then $|N \backslash Z| \geq(p-1)|N \cap Z|$.

Proof. It is trivial to show that if $u \in N \cap Z$ and $v \in N$, then $u+v \in N$. Therefore, if we take $u \in N \backslash Z$, the sets $i u+N \cap Z, i=1,2, \ldots, p-1$, are $p-1$ pairwise disjoint subsets of $N \backslash Z$, each of cardinality $|N \cap Z|$.

THEOREM 2.5. Let $R$ be a finite noncommutative ring, and write $R=S_{1} \oplus S_{2} \oplus \cdots \oplus$ $S_{k}$, where the $S_{i}$ 's are indecomposable. If $n$ of the $S_{i}$ 's are noncommutative and $p$ is the smallest prime dividing $|R|$, then $|N \backslash Z| \geq\left(p^{n}-1\right)|N \cap Z|$.

Proof. Proceeding by induction, the case $\mathrm{n}=1$ is Theorem 2.4 , and for $n \geq 2$ we may write $R=R_{1} \oplus R_{2}$ with $R_{1}, R_{2}$ as in the proof of Theorem 2.3. For $i=1,2$, denote by $Z_{i}$ and $N_{i}$ the sets $Z\left(R_{i}\right)$ and $N\left(R_{i}\right)$, respectively. Clearly, $|N \cap Z|=\left|N_{1} \cap Z_{1}\right|\left|N_{2} \cap Z_{2}\right|$. Therefore,

$$
\begin{aligned}
|N \backslash Z| & =\left|N_{1} \backslash Z_{1}\right|\left|N_{2}\right|+\left|N_{1} \cap Z_{1}\right|\left|N_{2} \backslash Z_{2}\right| \\
& \geq\left(p^{n}-1\right)\left|N_{1} \cap Z_{1}\right| p\left|N_{2} \cap Z_{2}\right|+(p-1)\left|N_{1} \cap Z_{1}\right|\left|N_{2} \cap Z_{2}\right| \\
& =\left(p^{n+1}-1\right)\left|N_{1} \cap Z_{1}\right|\left|N_{2} \cap Z_{2}\right| \\
& =\left(p^{n+1}-1\right)|N \cap Z| .
\end{aligned}
$$


EXAMPLE 2.6. Let $R_{1}=\left\{\left[\begin{array}{ll}a & b \\ 0 & 0\end{array}\right] a, b \in \mathrm{GF}(p)\right\}$ and let $R=R_{1} \oplus R_{1}$. Then

$$
\begin{gathered}
\left|D\left(R_{1}\right) \backslash Z\left(R_{1}\right)\right|=\left(p^{2}-1\right)\left|D\left(R_{1}\right) \cap Z\left(R_{1}\right)\right|, \\
\left|N\left(R_{1}\right) \backslash Z\left(R_{1}\right)\right|=(p-1)\left|N\left(R_{1}\right) \cap Z\left(R_{1}\right)\right| ; \\
|N(R) \backslash Z(R)|=\left(p^{2}-1\right)|N(R) \cap Z(R)| .
\end{gathered}
$$

Thus, the bounds in Theorems 2.2, 2.4, and 2.5 are best possible.

3. Cardinality of $D \backslash Z$ for infinite noncommutative rings. For infinite noncommutative rings, it is possible to have $D \subseteq Z$. [6, Ex. 1.1] is such a ring in which $D=N$. We give a further example in which $D \neq N$.

EXAMPLE 3.1. Let $K=\mathbb{Z}[x, y, z] /\left(y^{2}, y z\right)$ and let $\delta$ be the derivation on $K$ defined by $\delta(x)=y, \delta(y)=0, \delta(z)=0$. Let $R$ be the skew polynomial ring $K[t ; \delta]$. The elements of $K$ may be regarded as polynomials of the form $f_{0}(x)+f_{1}(x) z+\cdots+$ $f_{n}(x) z^{n}+g(x) y$, where $f_{0}(x), \ldots, f_{n}(x), g(x) \in \mathbb{Z}[x]$. If we let $L$ be the set of such polynomials with $f_{0}(x)=0$, it may be shown that $D(R)=L[t ; \delta]=L(t) \subseteq Z(R)$. The details are similar to those in [6, Ex. 1.1] and hence may be omitted.

If $R$ is an infinite noncommutative ring with $D \backslash Z \neq \varnothing$, then $D \backslash Z$ must be infinite. In fact, the following stronger result holds.

THEOREM 3.2. Let $R$ be an infinite ring. Then $|S \backslash Z|=|D \backslash Z|$; and if $S \backslash Z$ is nonempty, then it is infinite.

The proof of this theorem requires a sequence of lemmas.

LEMMA 3.3. (i) If $R$ is any infinite ring with $D \neq\{0\}$, then $|S|=|R|$.

(ii) If $R$ is any infinite ring with $D \neq\{0\}$ and $|S \backslash Z|<|R|$, then $|Z|=|R|$.

Proof. (i) This follows from the proof of [7, Thm. 6].

(ii) Since $S=(S \backslash Z) \cup(S \cap Z)$ and $|S|=|R|>|S \backslash Z|$, we have $|S \cap Z|=|R|$ and hence $|Z|=|R|$.

For the next three lemmas, $R$ is always assumed to be an infinite ring with $D \neq\{0\}$ and $|S \backslash Z|<|R|$.

LEMMA 3.4. Let $V$ be an infinite additive subgroup of $R$ with $V \subseteq S$. If $|V|>|S \backslash Z|$, then $V \subseteq Z$ and $a+V \nsubseteq S$ for any $a \in R \backslash Z$.

Proof. From $V=(V \backslash Z) \biguplus(V \cap Z)$ and $|V|>|S \backslash Z| \geq|V \backslash Z|$, we obtain $|V \cap Z|=|V|$. Now, for any group, the cardinal number of the complement of a proper subgroup cannot be smaller than that of the group. It follows that $V=V \cap Z$, so $V \subseteq Z$. Hence, for $a \notin Z, a+V \subseteq R \backslash Z$; and since $|a+V|=|V|>|S \backslash Z|$, we obtain $a+V \nsubseteq S$.

LEMMA 3.5. If $a \in S \backslash Z$, then either $Z a$ is finite or $|Z a| \leq|S \backslash Z|$. In either case, $|A(a)|=|R|$ and $A(a) \subseteq Z$.

Proof. If $Z a$ is infinite, then by taking $V=Z a$ in Lemma 3.4 and noting that $a+$ $Z a \subseteq S$, we see that $|Z a| \leq|S \backslash Z|$. Since $|S \backslash Z|<|R|$ and (by Lemma 3.3(ii)) $|Z|=|R|$, we have $|Z a|<|Z|$ and this equality also holds if $Z a$ is finite. 
Now consider the additive map from $Z$ onto $Z a$ given by $z \longmapsto z a, z \in Z$. Its kernel is $A(a) \cap Z$; and since $Z$ is infinite and $|Z a|<|Z|$, we get $|A(a) \cap Z|=|Z|=|R|$ and hence $|A(a)|=|R|$. Another appeal to Lemma 3.4 gives $A(a) \subseteq Z$.

LEMмA 3.6. Let $R$ be an infinite ring with $D \neq\{0\}$ and $|S \backslash Z|<|R|$. Then $N \subseteq Z$ and $D=S$.

Proof. Let $a \in N \backslash\{0\}$, so that $a \in S$ and $a+A(a) \subseteq S$. If $a \notin Z$, then by Lemma 3.5 we have $A(a) \subseteq Z$ and $|A(a)|=|R|$, from which it follows that $a+A(a) \subseteq S \backslash Z$ and $|S \backslash Z| \geq|a+A(a)|=|A(a)|=|R|$, in contradiction of our original hypothesis. Thus, $N \subseteq Z$. It is straightforward to show that this inclusion implies $D=S$.

We now have enough background to prove Theorem 3.2.

Proof OF TheOrem 3.2. If $D=\{0\}$, the result is trivial. If $|S \backslash Z|=|R|$, then obviously $|D \backslash Z|=|R|$. If $D \neq\{0\}$ and $|S \backslash Z|<|R|$, then $D=S$ by Lemma 3.6. Thus, in all cases $|S \backslash Z|=|D \backslash Z|$.

Assume that $S \backslash Z$ is finite and nonempty, in which case $|S \backslash Z|<|R|$. Invoking Lemma 3.5, we see that if $a \in S \backslash Z$, then $Z a$ is finite and $A(a) \subseteq Z$, so that $A(a)$ has finite index in $Z$. Let $I=A(S \backslash Z)$, and let $B=A(I)$. Now $I$ is the intersection of finitely many subgroups of $Z$ of finite index, hence it has finite index in $Z$; and since $Z$ is infinite by Lemma 3.3(ii), $I \neq\{0\}$ and therefore $B \subseteq S$. If $B$ were infinite, taking $V=B$ in Lemma 3.4 would yield $B \subseteq Z$, which contradicts the fact that $S \backslash Z \subseteq B$. Hence $B$ is finite. By Lemma 3.6, $N \subseteq Z$. Hence, $N(B) \subseteq Z(B)$ and $B$ is commutative by Theorem $H_{2}$. Since $S \backslash Z \subseteq B$, we see that $S$ is multiplicatively commutative.

Now choose $a \in S \backslash Z$. Then $a \notin N$, the finiteness of $B$ guarantees that some power of $a$ is a nonzero idempotent $e$; and since $N \subseteq Z, e \in Z$. It follows that $R=e R \oplus$ $A(e)$; and since $S$ is commutative, both $e R$ and $A(e)$ are commutative and hence $R$ is commutative-a contradiction. Therefore, $S \backslash Z$ must be infinite.

We note in passing that Lemma 3.6 in conjunction with Theorem $\mathrm{H}_{2}$ yields the following generalization of [2, Thm. 3].

THEOREM 3.7. A periodic ring with $|S \backslash Z|<|R|$ is either finite or commutative.

A crucial distinction between infinite rings and finite rings is that in infinite rings, it is possible to have $0<|D \backslash Z|<|D \cap Z|$, which, as pointed out in [6], is equivalent (in infinite rings) to the condition that $|D \backslash Z|<|R|$. In fact, $|D \backslash Z|$ may be any infinite cardinal number less than or equal to $|R|$, as the following theorem shows.

THEOREM 3.8. Given any infinite cardinal numbers $\alpha, \beta$ with $\alpha \leq \beta$, there exists a ring $R$ with $|R|=\beta$ and $|D \backslash Z|=\alpha$.

Proof. If $\alpha=\beta$, we need only take $R=M_{2}(F)$, where $F$ is a field with $|F|=\beta$.

If $\alpha<\beta$, the following construction yields an example (cf. [6, Ex. 2.1]). Let $R_{1}$ be any noncommutative ring with $D\left(R_{1}\right) \subseteq Z\left(R_{1}\right)$ and $\left|R_{1}\right|=\alpha$. (Such rings may be shown to exist by replacing $\mathbb{Z}[x, y, z]$ by $F[x, y, z]$ in Example 3.1, where $F$ is a field with $|F|=\alpha$.) Let $R_{2}$ be a field of cardinality $\beta$. Define $R$ to be $R_{1} \oplus R_{2}$. It is immediate that $|R|=\beta$ and it can be easily shown that $D(R) \backslash Z(R)$ is the set of noncentral elements of $R_{1}$. Now by Lemma 3.3(ii), $\left|Z\left(R_{1}\right)\right|=\alpha$; and since $\left|R_{1} \backslash Z\left(R_{1}\right)\right| \geq\left|Z\left(R_{1}\right)\right|$, we have 
$|D(R) \backslash Z(R)|=\alpha$ as required.

We remark that the condition that $0<|D \backslash Z|<|D \cap Z|$ imposes fairly strong structural conditions on $R$. [6, Thms. 2.12, 2.13, and 2.16] provide structural characterizations of such rings.

4. Some results involving nonnilpotent zero divisors. Theorem $H_{1}$ can be improved in another direction. We show that if the finite noncommutative ring $R$ has $D \neq N$, then $(D \backslash N) \backslash Z \neq \varnothing$; and if $S \neq N$, then $(S \backslash N) \backslash Z \neq \varnothing$.

In fact, our discussion goes well beyond the case of finite rings. The fundamental tool is the following lemma.

LEMMA 4.1. (i) If $R$ is a ring with $D \neq N$ and $D \backslash N \subseteq Z$, then $N \subseteq Z$.

(ii) If $R$ is a ring with $S \neq N$ and $S \backslash N \subseteq Z$, then $N \subseteq Z$.

Proof. (i) Let $a \in N$ and $b \in D \backslash N$. Then $a+b \in D \backslash N$ by Lemma 1.1(i). Thus, $a=a+b-b \in Z$.

(ii) Use the same argument, but with Lemma 1.1(ii).

Using this lemma and Theorem $\mathrm{H}_{2}$, we obtain the following theorem, which includes the results for finite rings which we announced at the beginning of this section.

THEOREM 4.2. Let $R$ be a periodic ring in which $D \neq N$ (resp., $S \neq N$ ). If $D \backslash N \subseteq Z$ (resp., $S \backslash N \subseteq Z$ ), then $R$ is commutative.

This result can be extended to larger classes of rings. Call a ring $R$ weakly periodic if $R=P+N$, where $P$ is the set of potent elements, i.e., the set of $x \in R$ for which there exists an integer $n=n(x)>1$ such that $x^{n}=x$. Call $R$ quasi-periodic if for each $x \in R$, there exist integers $n, m, k$ with $n>m>0$ such that $x^{n}=k x^{m}$. The classes of weakly periodic rings and quasi-periodic rings both contain the class of periodic rings. The containment is proper in the case of quasi-periodic rings (for example, $\mathbb{Z}$ is quasi-periodic). Whether the containment is proper for weakly periodic rings is not known.

It was proved in [3] that every weakly periodic ring with $N$ commutative is periodica result which, in conjunction with Theorem $\mathrm{H}_{2}$, shows that a weakly periodic ring with $N \subseteq Z$ must be commutative. It was proved in [1] that every quasi-periodic ring with $N \subseteq Z$ is commutative. Thus, our lemma yields

THEOREM 4.3. Let $R$ be a ring which is either weakly periodic or quasi-periodic. If $D \neq N($ resp., $S \neq N$ ) and $D \backslash N \subseteq Z$ (resp., $S \backslash N \subseteq Z$ ), then $R$ is commutative.

To conclude the paper, we return to the case of finite rings and present a theorem analogous to Theorem 2.1.

THEOREM 4.4. Let $R$ be a finite indecomposable noncommutative ring in which $|R|$ is a power of the prime $p$. If $D \neq N$, then $|(D \backslash N) \backslash Z| \geq\left(p^{2}-p\right)|D \cap Z|$.

Proof. We return to the case $D \neq N$ in the proof of Theorem 2.1. (Note that while this case appears under the assumption that $R \neq D$, the argument also works if $R=D$.) Let $e, f$, and $u$ be as before. 
We showed that for $i, j=0,1,2, \ldots, p-1$ and $(i, j) \neq(0,0)$, the set $i e+j f+D \cap Z \subseteq$ $D \backslash Z$; and we now determine which of these sets consist of nonnilpotent elements.

Let $x=i e+j f$. Since $D \cap Z \subseteq N$, we see that $(x+D \cap Z) \cap N \neq \varnothing$ if and only if $x \in N$. But $x=(i+j) e+j u$; and since $e u=u$ and $u e=u^{2}=0$, we have $x^{m}=$ $(i+j)^{m} e+(i+j)^{m-1} j u$ for all positive integers $m$. Thus, $x \in N$ if and only if $i+j \equiv 0$ $(\bmod p)$, and the theorem follows immediately.

We note, in conclusion, that Theorem 4.4 is best possible; the ring $R_{1}$ of Example 2.6 has $|(D \backslash N) \backslash Z|=\left(p^{2}-p\right)|D \cap Z|$.

ACKNOWLEDGEMENT. Dr. Bell's research was supported by the Natural Sciences and Engineering Research Council of Canada.

\section{REFERENCES}

[1] H. E. Bell, On quasi-periodic rings, Arch. Math. (Basel) 36 (1981), no. 6, 502-509. MR 83a:16042. Zbl 457.16010.

[2] _ Some conditions for finiteness of a ring, Internat. J. Math. Math. Sci. 11 (1988), no. 2, 239-242. MR 89c:16025. Zbl 647.16015.

[3] H. E. Bell and H. Tominaga, On periodic rings and related rings, Math. J. Okayama Univ. 28 (1986), 101-103. MR 88c:16029. Zbl 688.16022.

[4] I. N. Herstein, A proof of a conjecture of Vandiver, Proc. Amer. Math. Soc. 1 (1950), 370-371. MR 12,75h. Zbl 040.01302.

[5] _ A note on rings with central nilpotent elements, Proc. Amer. Math. Soc. 5 (1954), 620. MR 16,5c. Zbl 055.26003.

[6] A. A. Klein and H. E. Bell, On central and noncentral zero divisors, 26 (1998), no. 4, 1277 1292.

[7] C. Lanski, Rings with few nilpotents, Houston J. Math. 18 (1992), no. 4, 577-590. MR 94c:16026. Zbl 821.16020.

[8] D. L. Outcalt and A. Yaqub, A commutativity theorem for rings, Bull. Austral. Math. Soc. 2 (1970), 95-99. MR 41\#3511.

BelL: DePARTMENT OF MATHEMATICS, BRock UNIVERSity, ST. CATHARINES, ONTARIO L2S 3A1, CANADA

E-mail address: hbe11@spartan.ac.brocku.ca

KLein: SaCkLeR Faculty of EXact Sciences, School of Mathematical Sciences, Tel-AViv UNIVERSITY, TEL-AVIV 69978, ISRAEL

E-mail address: aak1ein@math. tau.ac. $i 1$ 


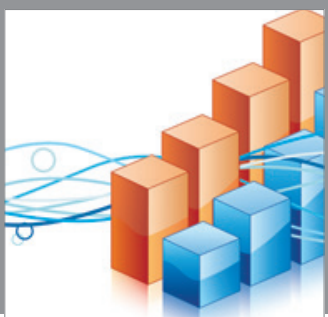

Advances in

Operations Research

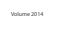

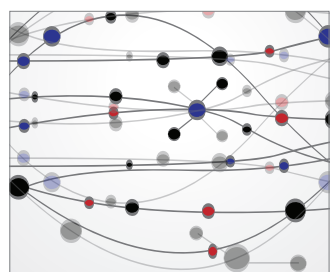

\section{The Scientific} World Journal
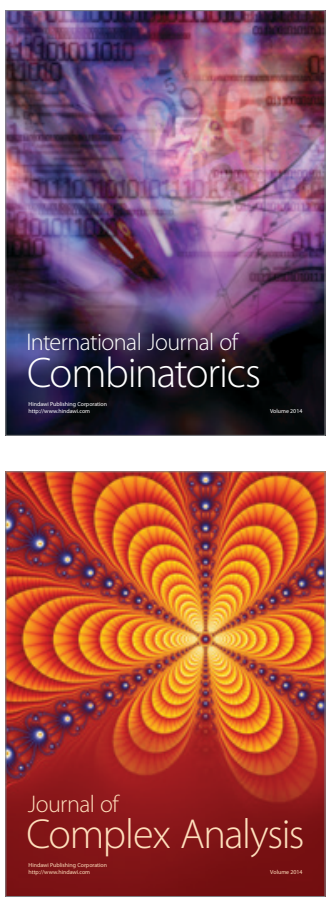

International Journal of

Mathematics and

Mathematical

Sciences
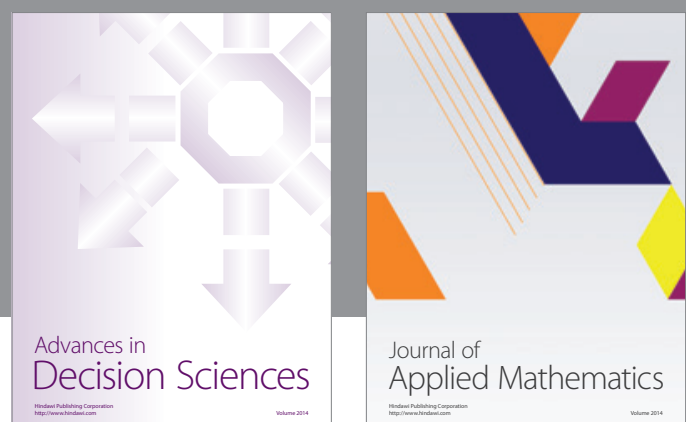

Journal of

Applied Mathematics
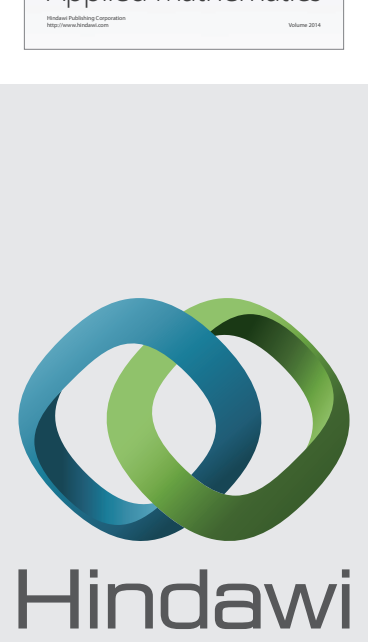

Submit your manuscripts at http://www.hindawi.com
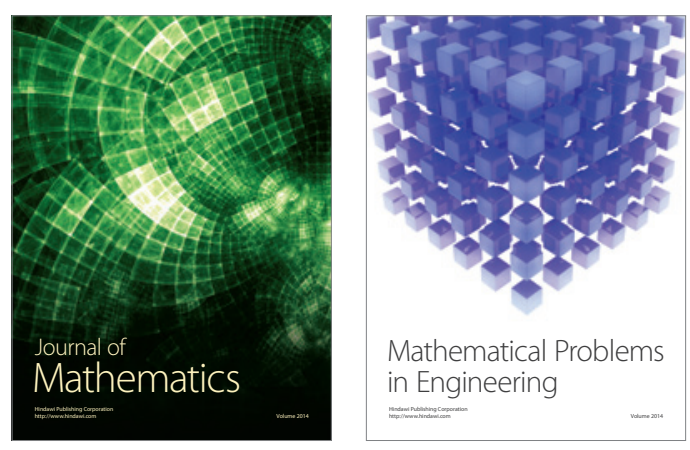

Mathematical Problems in Engineering
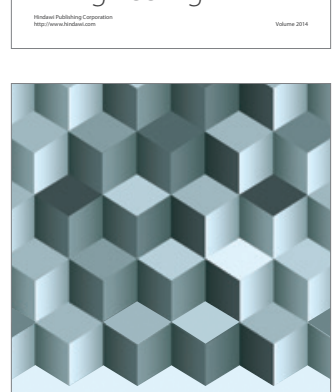

Journal of

Function Spaces
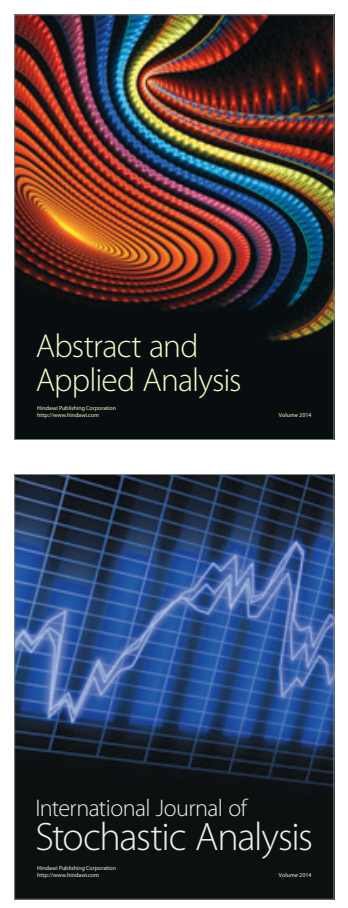

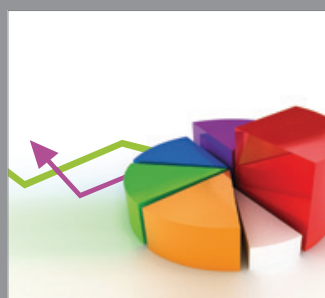

ournal of

Probability and Statistics

Promensencen
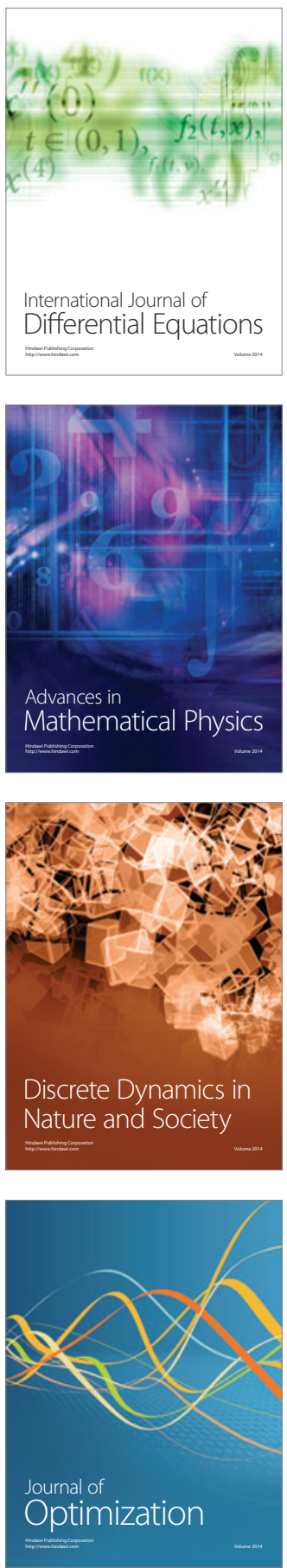